\title{
Receding Horizon Pseudospectral Control for Energy Maximization With Application to Wave Energy Devices
}

\author{
Romain Genest and John V. Ringwood, Senior Member, IEEE
}

\begin{abstract}
This paper addresses the issue of real-time control for applications, subject to physical constraints, involving an energy maximization objective. Typical application areas include renewable energy systems where, in spite of the fact that the raw energy resource is free, the capital and operational costs associated with the energy conversion process are not. In addition, economic energy delivery can only be achieved if the conversion device is operated efficiently. Previous approaches to this problem include model predictive control (MPC), but the computational cost associated with MPC can be high. Pseudospectral solutions show considerable promise in achieving a good balance between performance and computation, but currently available solutions deal with fixed-period optimization. In this paper, a receding horizon real-time pseudospectral control is developed, based on half-range Chebyshev Fourier basis functions, which can accurately represent harmonic signals in the application domain, while also efficiently dealing with the signal truncation effects associated with a receding horizon formulation. An application example, based on a wave energy converter, is used to illustrate the new control algorithm.
\end{abstract}

Index Terms-Optimal control, pseudospectral method, receding horizon, wave energy.

\section{INTRODUCTION}

$\mathbf{T}$ $\mathrm{HE}$ problem addressed in this paper is a real-time receding horizon control design for constrained control problems where the performance function is an energy function. Such problems are becoming common in renewable energy systems, such as wave energy [1], tidal stream energy [2], wind energy [3], or combination of renewable energies [4], where the resource is ostensibly free, but the capital and maintenance costs are not [5]. Renewable energy costs [6] are still in excess of many conventional energy sources such as fossil fuels and, as a result, many jurisdictions offer favorable feed-in tariffs to encourage their development. However, such uneconomic feed-in tariffs are likely to diminish with time in order to preserve moderate energy prices as increasing amounts of renewable energy becomes available. In addition, the intermittency of renewable energy

Manuscript received August 13, 2015; revised January 19, 2016; accepted April 3, 2016. Date of publication May 6, 2016; date of current version December 14, 2016. Manuscript received in final form April 11, 2016. This work was supported by the Science Foundation Ireland under Grant SFI/13/IA/1886. Recommended by Associate Editor A. Alessandri.

The authors are with the Centre for Ocean Energy Research, Maynooth University, Maynooth, County Kildare, Ireland (e-mail: rgenest@eeng.nuim.ie; john.ringwood@eeng.nuim.ie).

Color versions of one or more of the figures in this paper are available online at http://ieeexplore.ieee.org.

Digital Object Identifier 10.1109/TCST.2016.2554524 sources increases the cost of renewables to the transmission system operator, compared with dispatchable conventional sources.

One of the key technological contributors to reducing the cost of renewable energy is control systems technology [7]-[9]. With a fixed level of capital investment, it is imperative that maximum energy is converted by the renewable energy converter, mindful of the need to keep the system operating within safe constraints. While MPC and other control strategies have been applied to wind turbines, the development of control strategies for tidal turbines and wave energy devices is at a relatively early stage. In addition, the high computational requirements of MPC can make real-time implementation challenging.

Control algorithms based on spectral methods offer an interesting alternative to MPC, as they can be used to solve optimal control problems under constraints using a specific parameterization of the solution [10]. Spectral methods have shown promise in computational aspects, offering the possibility of scaling in complexity/performance by changing the number of approximating basis functions. However, to date, only single period pseudospectral solutions have been presented in the renewable energy field [11], [12]. Recent studies have shown the utility of developing a receding horizon control to solve optimal control problem in real time such as [13] and [14]. This paper proposes an application of pseudospectral methods for receding horizon real-time control, based on half-range Chebyshev-Fourier (HRCF) basis functions, introduced in [15].

HRCF are particularly well suited to problems where the system variables are described by harmonic functions, permitting the determination of both the transient and steady-state components of the optimal trajectory, and allowing real-time application. Furthermore, HRCF functions, giving a Fourier extension for nonperiodic signals, are especially suited for the wave energy field, since wave elevation and fluid-structure interaction forces are all well described using Fourier analysis. Solar and wind energies involve state and control variables that could be well described using HRCF functions; despite their stochastic behavior, external forces are often low-pass filtered and exhibit an oscillatory behavior. This method does not require the use of cutoff functions, such as employed in [16], to generate periodic functions for a direct Fourier analysis and does not suffer from the Gibbs phenomenon while generating a Fourier series. As a case study, this paper demonstrates 
a worked example for solving a wave energy control problem for a particular type of wave energy converter (WEC).

\section{Generic Control Problem Description AND SOLUTION OUTLINE}

\section{A. Problem Specification}

The optimal control solution for renewable energy devices typically entails an energy production maximization over a given time horizon and under dynamical constraints dictating the allowable system behavior. Consideration of physical limitations are also essential in the design of a realistic control algorithm and imply additional inequality constraints in the problem formulation. The cost function is defined by (2) and depends on the state and control functions, respectively, $\mathbf{x}$ and $u$ of the considered system. The time horizon under which the absorbed energy is maximized is $\left[t_{0}, t_{0}+T\right]$, where $t_{0} \in \mathbb{R}^{+}$corresponds to the initial time and $T \in \mathbb{R}^{+}$is the prediction time horizon. This prediction time horizon corresponds to the time window where the energy resource can be forecasted with an acceptable accuracy for use in a pseudospectral optimal control problem formulation. The interval $\left[t_{0}, t_{0}+T\right]$ is mapped, for simplicity, into $[-1,1]$, using the following affine transformation:

$$
\tau=\frac{2}{T}\left(t-t_{0}\right)-1
$$

where $t \in\left[t_{0}, t_{0}+T\right]$ and $\tau \in[-1,1]$. We consider the following finite-horizon and constrained optimal control problem:

$$
\begin{cases}\max & J=\int_{-1}^{1} F(\mathbf{x}(\tau), u(\tau), \tau) d \tau \\ \text { s.t. } & \dot{\mathbf{x}}(\tau)=\mathbf{f}(\mathbf{x}, u, \tau), \quad \tau \in[-1,1] \\ & \mathbf{x}(-1)=\mathbf{x}_{0} \\ & \mathbf{H}(\mathbf{x}, u) \leq 0, \quad \tau \in[-1,1]\end{cases}
$$

where $F$ corresponds to the absorbed power, $\mathbf{H}$ corresponds to inequality constraints, and $\mathbf{f}$ represents the device dynamics.

\section{B. Control Solution Outline}

1) Pseudospectral Method: The method of weighted residual (MWR) is widely used in various engineering applications to solve boundary value or eigenvalue problems [17] by expressing the approximated solution in a finite subspace of a Hilbert space. The MWR can also be used for optimal control problems involving the minimization, or maximization, of a particular cost function while insuring that the state and control variables respect a specific system of dynamical equations and inequality constraints [18]. The MWR derives an approximate solution for optimal control problems, insuring equality and inequality constraints by annulment of the residuals. Projections of the residuals on a finite-dimensional space, spanned by a particular set of orthogonal functions, called trial functions, are cancelled. Unlike MPC, which effectively uses local zero-order holder ( $\mathrm{ZOH})$ functions to approximate the optimal solution, spectral methods are generally based on global functions defined over the complete control horizon. Various basis functions can be chosen to obtain an approximation of the optimal solution, and the choice of a particular basis is discussed in Section II-B2. The choice of trial functions dictates the type of spectral method use, for example, the Galerkin method [19], or collocation methods [20].

The cost function minimization, or maximization, is realized using nonlinear programming methods [21], such as sequential quadratic program algorithms, leading to interesting computational aspects since the solution is sought in a finite-dimensional vector space.

2) Function Approximation: Pseudospectral methods are based on an approximation of the state and control variables into an $N$-D vector space $E$ generated by an orthogonal basis of real functions $B=\left\{\phi_{1}, \ldots, \phi_{N}\right\}$. This approximation is usually realized using interpolation methods or truncated generalized Fourier series. Control and state variables are commonly rewritten as

$$
\begin{aligned}
& x_{i} \simeq x_{i}^{N}=\sum_{k=1}^{N} \phi_{k} x_{i k}=\Phi X_{i} \\
& u \simeq u^{N}=\sum_{k=1}^{N} \phi_{k} u_{k}=\Phi U
\end{aligned}
$$

with $\Phi=\left[\phi_{1}, \ldots, \phi_{N}\right], \quad X_{i}=\left[x_{i 1}, \ldots, x_{i N}\right]^{T}$, and $U=\left[u_{1}, \ldots, u_{N}\right]^{T}$. A wide variety of basis functions can be used to approximate the state and control variables and the choice of a particular orthogonal base is mainly dictated by the specific requirements of the control problem.

Orthogonal wavelets are a large family of functions used for a wide variety of applications. For instance, Haar wavelets can be employed to solve nonlinear optimal control problems [22], Legendre wavelets constitute appropriate candidates for pseudospectral method basis sets for the resolution of various boundary value problems [23], and other wavelet families, such as Morlet wavelet, are widely used in signal processing in diversified domains [24]. Orthogonal wavelet bases are typically generated through a scaling and shifting process leading to a significant number of derived basis functions.

Truncated Fourier series give satisfying approximations for relatively smooth functions and have been employed in pseudospectral optimal control, including in the wave energy field [11]. Fourier approximations are particularly well adapted to wave signal approximations and constitute a good first choice for pseudospectral resolution. For finite-time horizon control, the Fourier basis requires periodicity of the approximated functions in order to avoid the Gibbs phenomenon on the boundaries. Since the Fourier basis generates only periodic functions, different boundary values of the approximated functions lead to discontinuities and higher frequency harmonics are needed to obtain a correct approximation. A solution has been proposed in [16] to avoid such discontinuities by adding a buffer polynomial to construct an extended periodic function after which a standard Fourier pseudospectral method is applied.

Lagrange polynomials are commonly used in Legendre methods [25], Chebyshev methods [26], or more generally in Jacobi methods [27] to interpolate or approximate the control and state variables. A certain amount of precaution has to be 
(a)

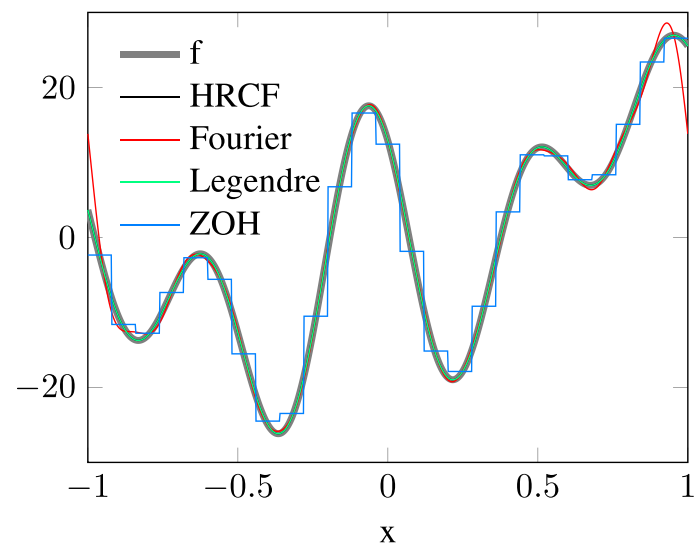

(b)

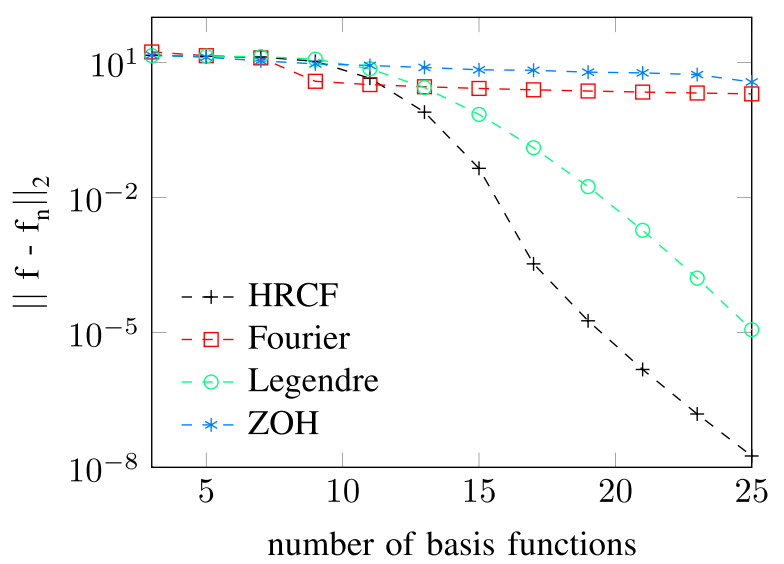

Fig. 1. Approximation of a nonperiodic function using different sets of functions. (a) Approximation errors for various orthgonal function sets. (b) Approximation of the function $\mathrm{f}$ using various orthogonal function sets.

taken in the choice of the collocation points in order to avoid the Runge phenomenon [28] during the interpolation.

In sustainable energy, particularly for WECs optimal control problems, Fourier-type bases seem to be a straightforward choice, since the wave elevation, excitation force, and radiation force are usually well described via Fourier analysis. However, real-time control and finite-time control horizons require approximations of nonperiodic functions in order to simulate both the transient and steady-state responses of the device. A combination of the real-time and finite-time horizon requirements suggests a basis employing HRCF functions, originally defined in [15] and presented in more detail in Section III.

By a way of example, Fig. 1 shows a comparison between the approximations of a nonperiodic function defined on $[-1,1]$ using different sets of basis functions. As an illustrative example, the approximated function presented in Fig. 1 is a sum of $N_{S}$ sine functions with random amplitudes, phases, and periods uniformly distributed between, respectively, the following intervals $[0,1],[0,2 \pi]$, and $[0.5,2] ; N_{S}$ is arbitrarily set to $10^{3}$. Fig. 1(a) shows the approximation obtained with 25 functions such as $\mathrm{ZOH}$ functions or Haar wavelets, truncated Fourier series, Legendre polynomials, and HRCF functions. Legendre polynomials and $\mathrm{HRCF}$ functions give

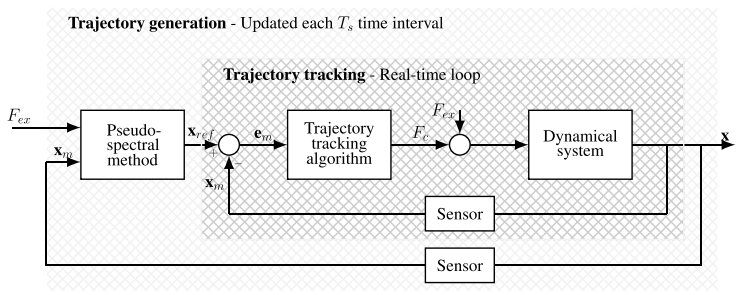

Fig. 2. Block diagram of the control algorithm.

almost identical results, and the differences between the initial function and their truncated generalized Fourier series are indistinguishable. However, truncated Fourier series and $\mathrm{ZOH}$ functions do not provide such a satisfactory approximation for the example in Fig. 1. Using Fourier series to approximate nonperiodic functions leads to boundary discontinuities and the emergence of the Gibbs phenomenon. Haar wavelets or $\mathrm{ZOH}$ functions both need a large number of basis functions to attain the same level of polynomial or HRCF approximation error. As a result, a large number of $\mathrm{ZOH}$ functions have to be utilized, increasing the computational time for an optimal control problem using a standard MPC algorithm such as [29].

3) Solution Route Outline: The method proposed in this paper comprises a hierarchically organized upper and lower loop, which, respectively, generate and track a reference trajectory as shown in Fig. 2. The upper loop determines the reference trajectory using a pseudospectral method with HRCF basis functions solved by a nonlinear programming method, such as sequential quadratic programming (SQP) algorithm [30] implemented in MATLAB via the fmincon function. The lower loop facilitates tracking of the reference trajectory in real time. Trajectory generation is updated at a regular interval $T_{S}$ and tracked using a standard backstepping method [31]. The complete structure of the control algorithm is presented in Fig. 2.

The remainder of this paper is organized as follows. Based on [15], [32], and [33], the definition and application of the HRCF basis for pseudospectral methods is presented in Section III. A direct application of the control algorithm for a WEC is presented in IV. Pseudospectral method using HRCF functions applied to a generic WEC with $1 \mathrm{DOF}$ is presented in Sections IV-A and IV-B, while the backstepping method used for the lower loop, to track the reference trajectory, is described in Section IV-C. Finally, the results are given in Section IV-E for a particular WEC controlled in real time under constraints and subject to irregular waves.

\section{Half-RAnge Chebyshev Fourier Functions}

\section{A. Half-Range Chebyshev Polynomials}

HRCF functions were introduced in 2010 [15] offering a new alternative in the approximation of nonperiodic functions. An orthogonal basis for nonperiodic functions is determined based on half-range Chebyshev polynomials of the first and the second kind. Half-range Chebyshev polynomials of the first and the second kind of order $k, T_{k}^{h}$ and $U_{k}^{h}$, respectively, are orthogonal with lower order monomials with respect to the weights $1 /\left(1-y^{2}\right)^{1 / 2}$, for the first kind, and $\left(1-y^{2}\right)^{1 / 2}$, for the second kind, on the interval $[0,1]$. Definitions of $T_{k}^{h}$ and $U_{k}^{h}$ are given in [32] based on [15]. 

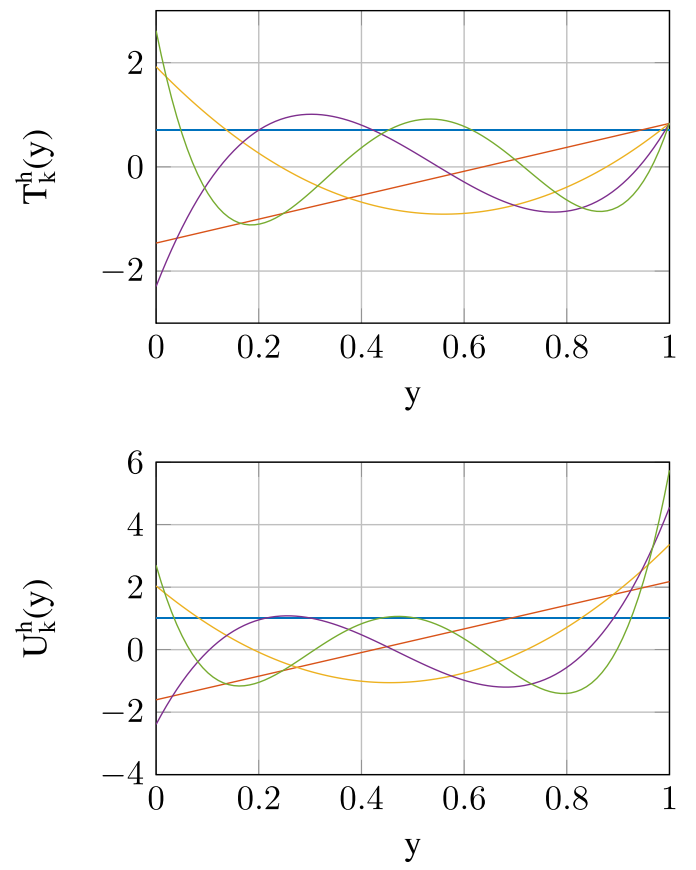

Fig. 3. Half-range Chebyshev polynomials of the first and the second kind.

Definition 1: Let $T_{k}^{h}(y)$ be the unique normalized sequence of orthogonal polynomials satisfying

$$
\begin{aligned}
& \int_{0}^{1} T_{k}^{h}(y) y^{l} \frac{1}{\sqrt{1-y^{2}}} d y=0, \quad l=0, \ldots, k-1 \\
& \frac{4}{\pi} \int_{0}^{1} T_{k}^{h}(y)^{2} \frac{1}{\sqrt{1-y^{2}}} d y=1 .
\end{aligned}
$$

The set $\left\{T_{k}^{h}(y)\right\}_{k=0}^{\infty}$ is a set of half-range Chebyshev polynomials of the first kind.

Definition 2: Let $U_{k}^{h}(y)$ be the unique normalized sequence of orthogonal polynomials satisfying

$$
\begin{aligned}
& \int_{0}^{1} U_{k}^{h}(y) y^{l} \sqrt{1-y^{2}} d y=0, \quad l=0, \ldots, k-1 \\
& \frac{4}{\pi} \int_{0}^{1} U_{k}^{h}(y)^{2} \sqrt{1-y^{2}} d y=1 .
\end{aligned}
$$

The set $\left\{U_{k}^{h}(y)\right\}_{k=0}^{\infty}$ is a set of half-range Chebyshev polynomials of the second kind. Half-range Chebyshev polynomials of the first and second kind are shown in Fig. 3.

\section{B. Solution to the Approximation Function Problem}

One way to obtain the Fourier series of any nonperiodic function $f \in L_{[-1,1]}^{2}$ is to extend $f$ to a periodic function $g$ defined in a larger interval in this case $[-2,2]$. Determination of the Fourier extension of $f$ can be stated as an optimization problem.

Problem 1: Let $G_{n}$ be the space of four-periodic functions of the form

$$
g \in G_{n}: g(\tau)=\frac{a_{0}}{2}+\sum_{k=1}^{n} a_{k} \cos \frac{\pi}{2} k \tau \cdots+b_{k} \sin \frac{\pi}{2} k \tau .
$$

The Fourier extension of $f$ to the interval $[-2,2]$ is the solution to the optimization problem

$$
g_{n}:=\arg \min _{g \in G_{n}}\|f-g\|_{L_{[-1,1]}^{2}} .
$$

Huybrechs [15] proved the existence and uniqueness of the solution of problem 1 based on orthogonal polynomials called HRCF polynomials. Two sets $\left\{T_{k}^{h}(\cos (\pi / 2) \tau)\right\}_{k=0}^{n}$ and $\left\{U_{k}^{h}(\cos (\pi / 2) \tau) \sin (\pi / 2) \tau\right\}_{k=0}^{n-1}$ are introduced, constituting an orthonormal basis for, respectively, the $(n+1)$ - and $n$-D spaces spanned by the four-periodic, respectively, cosine and sine functions. The exact solution of problem 1 is thus directly found by an orthogonal projection of $f$, as expressed in the following:

$$
\begin{aligned}
g_{n}(\tau)= & \sum_{k=0}^{n} a_{k} T_{k}^{h}\left(\cos \frac{\pi}{2} \tau\right) \ldots \\
& +\sum_{k=0}^{n-1} b_{k} U_{k}^{h}\left(\cos \frac{\pi}{2} \tau\right) \sin \frac{\pi}{2} \tau
\end{aligned}
$$

where

$$
a_{k}=\int_{-1}^{1} f(\tau) T_{k}^{h}\left(\cos \frac{\pi}{2} \tau\right) d \tau
$$

and

$$
b_{k}=\int_{-1}^{1} f(\tau) U_{k}^{h}\left(\cos \frac{\pi}{2} \tau\right) \sin \frac{\pi}{2} \tau d \tau .
$$

As was shown in [32], the sets of basis functions $\left\{T_{k}^{h}(\cos (\pi / 2) \tau)\right\}_{k=0}^{n}$ and $\left\{U_{k}^{h}(\cos (\pi / 2) \tau) \sin (\pi / 2) \tau\right\}_{k=0}^{n-1}$ can be employed in the resolution of a nonperiodic boundary value problem using pseudospectral methods.

\section{Computation With Half-Range Chebyshev Polynomials}

1) Differentiation Matrix: Based on the HRCF functions, Orel and Perne [33] developed in an efficient calculation of the derivatives of truncated HRCF series. Let $g_{n}$ be the truncated HRCF series of a function $f \in L_{[-1,1]}^{2}$, so that

$$
\begin{aligned}
f(\tau) \simeq g_{n}(\tau)= & \sum_{k=0}^{n} a_{k} T_{k}^{h}\left(\cos \frac{\pi}{2} \tau\right) \ldots \\
& +\sum_{k=0}^{n-1} b_{k} U_{k}^{n}\left(\cos \frac{\pi}{2} \tau\right) \sin \frac{\pi}{2} \tau .
\end{aligned}
$$

The derivative of $g_{n}$ is then defined by the following:

$$
\begin{aligned}
\frac{d g_{n}}{d \tau}= & \sum_{k=0}^{n} a_{k}^{\prime} T_{k}^{h}\left(\cos \frac{\pi}{2} \tau\right) \ldots \\
& +\sum_{k=0}^{n-1} b_{k}^{\prime} U_{k}^{n}\left(\cos \frac{\pi}{2} \tau\right) \sin \frac{\pi}{2} \tau .
\end{aligned}
$$

In matrix form, $D \in \mathbb{R}^{(2 n+1) \times(2 n+1)}$, defined in [33], is such tha $\mathbf{f}^{\prime}=D \mathbf{f}$, where $\mathbf{f}=\left[a_{0}, \ldots, a_{n}, b_{0}, \ldots, b_{n-1}\right]^{T}$ and $\mathbf{f}^{\prime}=\left[a_{0}^{\prime}, \ldots, a_{n}^{\prime}, b_{0}^{\prime}, \ldots, b_{n-1}^{\prime}\right]$. The differentiation matrix $D$ is written in the following form:

$$
D=\frac{\pi}{2}\left(\begin{array}{cc}
0 & H_{1} \\
H_{2} & 0
\end{array}\right)
$$


where the matrices $H_{1} \in \mathbb{R}^{(n+1) \times n}$ and $H_{2} \in \mathbb{R}^{n \times(n+1)}$ are not expanded here for brevity; the reader may refer directly to [33] for a more complete exposition.

2) Function Multiplication: In the same manner, Orel and Perne [33] present a matrix formulation to determine the truncated series coefficients of the product $p_{n}=g_{n} \cdot h_{n}$ of truncated series of an arbitrary function $f \in L_{[-1,1]}^{2}$ and a known function $h \in L_{[-1,1]}^{2}$. In matrix form, a matrix $F \in \mathbb{R}^{(2 n+1) \times(2 n+1)}$ is defined such that $\mathbf{p}=F \mathbf{f}$, where $\mathbf{p}=\left[a_{0}^{\prime}, \ldots, a_{n}^{\prime}, b_{0}^{\prime}, \ldots, b_{n-1}^{\prime}\right]^{T}$ and $\mathbf{f}=\left[a_{0}, \ldots\right.$, $\left.a_{n}, b_{0}, \ldots, b_{n-1}\right]^{T}$, with

$$
F=\left(\begin{array}{ll}
G_{1} & G_{2} \\
G_{3} & G_{4}
\end{array}\right)
$$

where the matrix $G_{1} \in \mathbb{R}^{(n+1) \times(n+1)}, G_{2} \in \mathbb{R}^{(n+1) \times n}$, $G_{3} \in \mathbb{R}^{n \times(n+1)}$, and $G_{4} \in \mathbb{R}^{n \times n}$ are functions of the coefficients of the truncated series $g_{n}$ that approximates the known function $h$ and are defined in [33].

3) Convolution Product: The convolution product between an arbitrary causal function $f \in L^{2}(\mathbb{R})$ and a known causal function $h \in L^{2}(\mathbb{R})$ is defined by the following:

$$
(f * h)(t)=\int_{0}^{t} f(s) \cdot h(t-s) d s \quad \forall t \in \mathbb{R}^{+} .
$$

Convolution products can occur in dynamical equations describing physical systems and an illustration is given in the chosen WEC application in Section IV where the fluidstructure interaction force, more specifically the radiation force, involves a convolution product with the radiation kernel function and the velocity of the floating body. The determination of the radiation force is generally achieved using various approximation methods, generally leading to a state-space model of the convolution product [34]. For example, Prony's method [35] approximates the radiation kernel function by a sum of complex exponentials, creating new state variables with their respective partial differential equations. Using such approximations will result in the unwelcome increase in the dimension of the function $\mathbf{f}(\mathbf{x}, u)$, taking into account new radiation state variables.

In this paper, a direct approximation of the radiation kernel function is achieved using the HRCF functions. The coefficients of the truncated HRCF series of the convolution product $\mathbf{c}=\left[a_{0}^{\prime}, \ldots, a_{n}^{\prime}, b_{0}^{\prime}, \ldots, b_{n-1}^{\prime}\right]^{T}$ between an arbitrary function $f \in L_{[-1,1]}^{2}$ and the known kernel function is given by

$$
\mathbf{c}=P \mathbf{f}
$$

where $\mathbf{f}=\left[a_{0}, \ldots, a_{n}, b_{0}, \ldots, b_{n-1}\right]^{T}$ is the vector of coefficients of the truncated HRCF series of $f$, and $P \in \mathbb{R}^{(2 n+1) \times(2 n+1)}$ is the convolution matrix depending on the kernel function of the convolution product. A direct application on hydrodynamic radiation convolution product is presented in Appendix.

\section{WaVe Energy DeVice CASE}

Since wave energy is struggling to become economical, due to high capital and operational costs and significant safety and durability requirements, adding device intelligence via embedded computing to boost the power production for a relatively small marginal cost constitutes a sensible approach. High-performance control of WECs can be instrumental in making wave energy harvesting economic [36]. A direct application is presented for energy production maximization of a specific flap-type WEC.

A wide variety of WEC devices have been designed and tested in recent years [37], based on bottom-referenced or self-reacting principle, in order to recover energy from the waves. Dynamical equations are used to describe the behavior of WECs in real sea conditions for either single or multibody devices and most of the studies, including this paper, are based on linearized fluid-structure interaction.

Optimal control of WEC requires knowledge of future values of the excitation force generated by the fluid onto the device's hull [38] and potentially requires the use of predictive algorithms [39]. The loss in the accuracy of predictive algorithms limits the prediction window for practical real-time usage [39]. Thus, updates have to be made to calculate future values of the wave excitation force experienced by the device's hull. A limited prediction horizon leads to a receding horizon type of control and implies a relatively short computation time, suggesting the choice of pseudospectral methods for the efficient calculation of the optimal reference trajectory.

Various wave energy control algorithms have been developed from simple philosophies, such as latching [40], linear damping, or reactive control [41], to more complex ones, such as MPC [42]. MPC gives promising results, since it allows high-performance levels to be reached while considering physical constraints, but suffers from significant computational demands. The real-time implementation of a model predictive controller for a WEC remains a complex and critical issue.

Real-time wave energy control algorithms deal with nonperiodic signals, since the prediction horizon is limited, and wave surface elevation, fluid-structure interaction forces, and body motion are usually described using spectral forms. The choice of HRCF functions appears to fit all the requirements needed to solve the WEC optimal control problem with pseudospectral method.

Furthermore, the exponential rate of convergence of the Fourier series of the extended periodic function has been already demonstrated [15] and offers a better rate of convergence than extensions realized with the use of cutoff functions [16].

\section{A. WEC Model}

For clarity, in this paper, we consider a WEC with only 1 DOF. The fluid is assumed to be inviscid and the flow incompressible, allowing the use of potential theory to determine fluid-structure interactions. The body displacement and the amplitude of the wave field are considered small enough to use linearized potential theory, leading to a linear equation of motion of the device, or Cummin's equation [43], defined by

$$
\left(I+I_{\infty}\right) \ddot{x}+\int_{0}^{t} K(t-s) \dot{x}(s) d s+S_{h} x=F_{\mathrm{ex}}+F_{c}
$$

where $x, \dot{x}$, and $\ddot{x}$ are the body displacement and its first and second derivatives, $F_{c}$ is the control force, $I$ is the mass 
or inertia of the body (depending on the type of degree of freedom considered), $I_{\infty}$ corresponds to the infinite frequency added mass, or inertia, of the device, $K$ is the kernel function for the radiation convolution product used to determine radiation forces from the velocity of the body, $S_{h}$ is the linearized hydrostatic stiffness, and $F_{\text {ex }}$ corresponds to the wave excitation force, or Froude-Krylov force, experienced by the device. Recent control approaches for nonlinear WEC models based on pseudospectral method is presented in [44].

From (20) and using the affine transformation $g:[-1,1] \rightarrow$ $\left[t_{0}, t_{0}+T\right], \tau \mapsto(T / 2)(\tau+1)+t_{0}$, we define the scaled excitation force $f_{\mathrm{ex}}=F_{\mathrm{ex}} \circ g$, the kernel function of the radiation convolution product $k=K \circ g$, position $x_{1}=x \circ g$, velocity $x_{2}=\dot{x} \circ g$, and control force $u=F_{c} \circ g$, leading to the following system of differential:

$$
\left\{\begin{array}{c}
\dot{x}_{2}=\frac{T}{2}\left(\begin{array}{l}
f_{\mathrm{ex}}+u \cdots-\int_{g^{-1}(0)}^{\tau} k\left(\tau-s+g^{-1}(0)\right) x_{2}(s) d s \ldots \\
\left.\quad-S_{h} x_{1}\right) /\left(I+I_{\infty}\right)
\end{array}\right. \\
\dot{x}_{1}=\frac{T}{2} x_{2}
\end{array}\right.
$$

where $g^{-1}(0)=-1-2 t_{0} / T$ is the antecedent of 0 from the affine transformation $g$. The system of differential (21) defines the equality constraints in the optimal control problem (2), specifying the function $\mathbf{f}$ of the state variable $\mathbf{x}=\left[x_{1}, x_{2}\right]^{T}$ and the control variable $u$ via the following equation:

$$
\dot{\mathbf{x}}=\left[\begin{array}{c}
\dot{x}_{1} \\
\dot{x}_{2}
\end{array}\right]=\mathbf{f}(\mathbf{x}, u, \tau), \quad \tau \in[-1,1] .
$$

Practical limitations are considered on the body motion, control force, and power output to ensure feasibility of the control and the safety and durability of the WEC in real sea conditions. For all $\tau \in[-1,1]$

$$
\left\{\begin{array}{l}
\left|x_{1}(\tau)\right| \leq X_{\max } \\
\left|x_{2}(\tau)\right| \leq V_{\max } \\
|u(\tau)| \leq U_{\max }
\end{array}\right.
$$

where $X_{\max }, V_{\max }$, and $U_{\max }$ are real positive constants corresponding to position, velocity, and control force limitations determined in advance to ensure feasibility and safety. Inequality constraints are rewritten in a matrix form using approximation functions, and $\forall \tau \in[-1,1]$

$$
\mathbf{H}(\mathbf{x}, u)=\left(\left[\begin{array}{c}
\Phi(\tau) \\
-\Phi(\tau)
\end{array}\right] \otimes I_{3}\right) \mathbf{X}-\left[\begin{array}{l}
1 \\
1
\end{array}\right] \otimes \mathbf{X}_{\max } \leq 0
$$

where $\mathbf{X}=\left[X_{1}^{T}, X_{2}^{T}, U^{T}\right]^{T}$ represents the projections of the state and control variables, $\mathbf{X}_{\max }=\left[X_{\max }, V_{\max }, U_{\max }\right]^{T}$ represents position, velocity, and control force limitations, $I_{3}$ is the 3-D identity matrix, and $\otimes$ denotes the Kronecker product. Equation (24) specifies the linear function $\mathbf{H}$ from the optimal control formulation in (2).

Finally, the cost function, representing the absorbed energy, is written using the approximation functions in the following form:

$$
J=-\int_{-1}^{1} U^{T} \Phi^{T}(\tau) \Phi(\tau) X_{2} d t
$$

The cost function $J$ represents the recovered energy by the control through the control force $u$ determined by integrating instantaneous absorbed power over the control horizon. In the case of orthonormal approximation functions, with respect to the inner product $\left\langle\phi_{i}, \phi_{j}\right\rangle=\int_{-1}^{1} \phi_{i}(\tau) \phi_{j}(\tau) d t$, the cost function described in (25) is simplified into the form $J=-U^{T} X_{2}$.

\section{B. Cancellation of the Residuals}

Replacing state variable derivatives terms and convolution terms coming from the determination of the radiation force in the expression of $\mathbf{f}$ from (21), one can express the two residual terms $r_{1}$ and $r_{2}$ as

$$
\left\{\begin{aligned}
r_{1}(\tau)= & \Phi(\tau)\left(\frac{2\left(I+I_{\infty}\right)}{T} D X_{2}+P X_{2} \cdots+S_{h} X_{1}-U\right) \\
& -f_{\mathrm{ex}}(\tau)+c_{0}(\tau) \\
r_{2}(\tau)= & \Phi(\tau)\left(X_{2}-\frac{2}{T} D X_{1}\right) .
\end{aligned}\right.
$$

Trial functions used by pseudospectral methods are Dirac distributions, leading to the cancellation of residual terms at particular instants, or collocation points. The interval $[-1,1]$ is covered by $N+1$ (corresponding to $2 n+1$ ) collocation points $\tau_{i}$, i.e., Chebyshev points of the second kind

$$
\tau_{i}=-\cos \left(\frac{\pi i}{N}\right), \quad i=0,1, \ldots, N .
$$

The cancellation of the first residual term $r_{1}$ at the collocation points leads to $N+1$ equations. The cancellation of the second residual term $r_{2}$ can be simplified, leading to $N+1$ equations linking the velocity and position projection vectors $X_{1}$ and $X_{2}$, as

$$
\left\{\begin{array}{l}
r_{1}\left(\tau_{i}\right)=0, \quad i=0,1, \ldots, N \\
X_{2}-\frac{2}{T} D X_{1}=0 .
\end{array}\right.
$$

This leads to $2 N+2$ dynamical equations determining, for a given projection vector $U$ of the control variable $u$, the $2 N+2$ variables composed of the $N+1$ components of $X_{1}$ and $X_{2}$.

\section{Trajectory Tracking}

Various control algorithms, such as PID [45], sliding control [46], or backstepping control [47], can be used to realize the tracking of the reference trajectory. For the WEC case study, the chosen lower control loop is based on a backstepping method and is described in the present section. The variables $x_{1}$ and $x_{2}$ refer, respectively, to the position and the velocity of the device, and the reference position and velocity trajectories are denoted, respectively, by $x_{1 \text {,ref }}$ and $x_{2, \text { ref. }} x_{1 \text {,ref }}$ and $x_{2, \text { ref }}$ are supposed to be known and determined previously by the upper control loop.

Let us define the function $V_{1}$ depending on the error $e_{1}=x_{1}-x_{1, \mathrm{ref}}$, as

$$
V_{1}=\frac{1}{2} e_{1}^{2}
$$




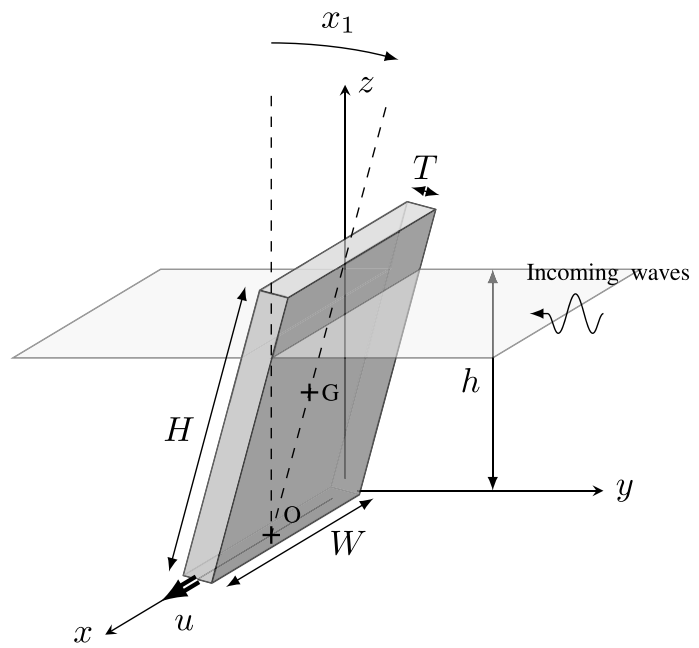

Fig. 4. Geometrical specification of the flap-type wave energy device.

In order for $V_{1}$ to be Lyapunov stable, we define the desired velocity $\dot{x}_{d}=x_{2, \text { ref }}-\tau_{1} e_{1}$ and the function $V_{2}$, as

$$
V_{2}=V_{1}+\frac{1}{2} e_{2}^{2}
$$

where $e_{2}=x_{2}-x_{2}$,ref. The derivative of $V_{2}$ is

$$
\dot{V}_{2}=-\tau_{1} e_{2}^{2}+e_{2}\left(e_{1}+\dot{x}_{2}-\ddot{x}_{d}\right) \text {. }
$$

Replacing the expression for the acceleration $\ddot{x}$ from Cummin's equation (20) in the derivative function $V_{2}$, we obtain

$$
\begin{aligned}
\dot{V}_{2}= & -\tau_{1} e_{1}^{2}+e_{2} \\
& \times\left(e_{1}+\frac{1}{I+I_{\infty}}\left(F_{\mathrm{ex}}+F_{c} \cdots-K * x_{2}-S_{h} x_{1}\right)-\ddot{x}_{d}\right)
\end{aligned}
$$

where $*$ denotes the convolution product. In order for $V_{2}$ to be Lyapunov stable, the control force $u$ has to be of the following form:

$$
F_{c}=-\left(I+I_{\infty}\right)\left(e_{1}-\ddot{x}_{d}+\tau_{2} e_{2}\right)-F_{\mathrm{ex}}+S_{h} x_{1}+K * x_{2} .
$$

\section{Specification of the WEC Model and Parameter Specification}

The control algorithm is tested on a flap-type WEC and its geometry is shown in Fig. 4. This generic WEC is based on the same working principle as the Oyster developed by Aquamarine Power Ltd [48], recovering wave energy from the oscillatory surge motion of a bottom-hinged vertical panel.

The density of the sea water is fixed at $\rho_{\text {water }}=1025 \mathrm{~kg} / \mathrm{m}^{3}$. The geometric characteristics are $W=30 \mathrm{~m}, T=1 \mathrm{~m}$, and $h=H=15 \mathrm{~m}$, the body density being assumed to be uniform and equal to $\rho_{\text {body }}=\rho_{\text {water }} / 8$. The total mass of the device is given by $m=\rho_{\text {body }} \times W \times T \times L \approx 58 \times 10^{3} \mathrm{~kg}$. The device inertia along the $x$-axis, expressed at the center of gravity, is $I_{G}=m\left(T^{2}+H^{2}\right) / 12$ and is expressed at the center of the pivot linkage $I=I_{G}+m(H / 2)^{2}$. The infinite frequency asymptote of the added inertia of the device,

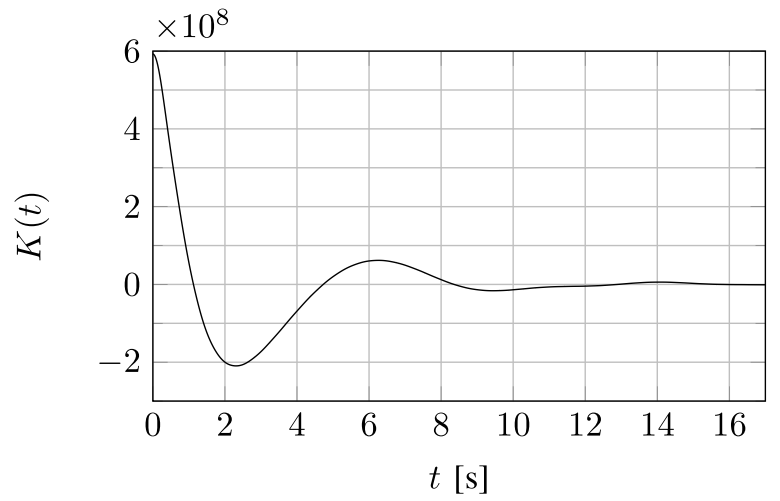

Fig. 5. Kernel function of the convolution product used to determine the radiation force.

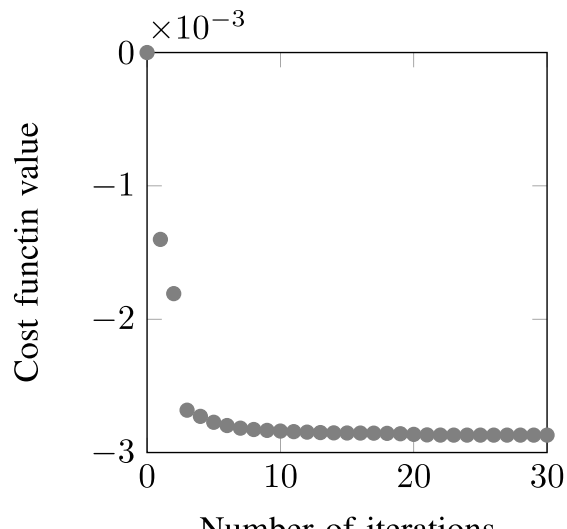

Fig. 6. Typical convergence curve of the SQP algorithm.

$I_{\infty}=2.5 \times 10^{8} \mathrm{kgm}^{2}$, and the kernel function $K$ of the convolution product used to model the radiation force are both determined using the potential code Aquaplus [49], with $K$ presented in Fig. 5 .

Irregular incident waves are considered, and the elevation of the free surface is determined using a Bretschneider spectrum [50], with a bandwidth of $2 \times 10^{-2} \mathrm{~Hz}$ to $5 \times 10^{-1} \mathrm{~Hz}$ and a frequency step $d f=5 \times 10^{-3} \mathrm{~Hz}$. The excitation force is determined using the Boundary Element Method code Aquaplus [49], based on the free surface elevation with randomly selected phases for the sinusoidal wave components.

The linear model is simulated in real time using a Runge-Kutta method with a time step of $\Delta t=10^{-2} \mathrm{~s}$. The prediction horizon, under which the optimization process is done, is chosen as $T=15 \mathrm{~s}$. The number of basis functions used to approximate the solution is $N+1=15$. The SQP algorithm is run for a maximum of 15 iterations, leading to an acceptable ratio between convergence quality and computational time, as shown in Fig. 6.

\section{E. Results}

The results are presented for a significant wave height of $H_{s}=1 \mathrm{~m}$ and a peak wave period of $T_{w}=8 \mathrm{~s}$, using a Bretschneider wave model (see Section IV-D). The computational time to solve one optimal control problem over the finite prediction horizon is less than $0.6 \mathrm{~s}$ for the complete 


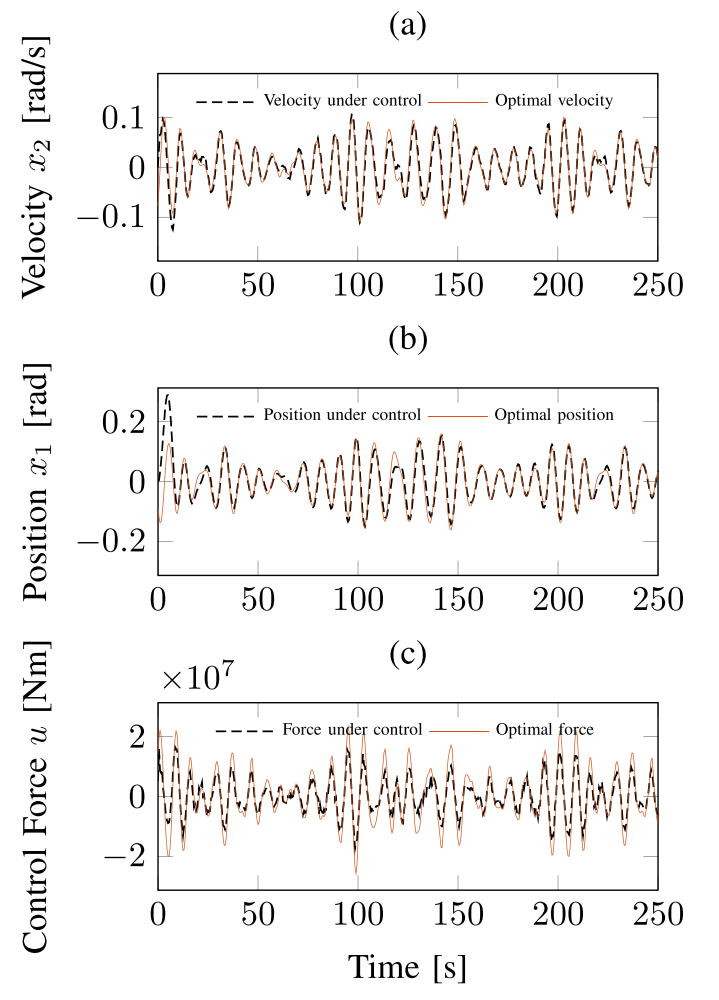

Fig. 7. Optimal trajectories (red solid line) and actual trajectories of the device under control (black dashed line). (a) Velocity, (b) position, and (c) control force for a 250 -s simulation.

(a)

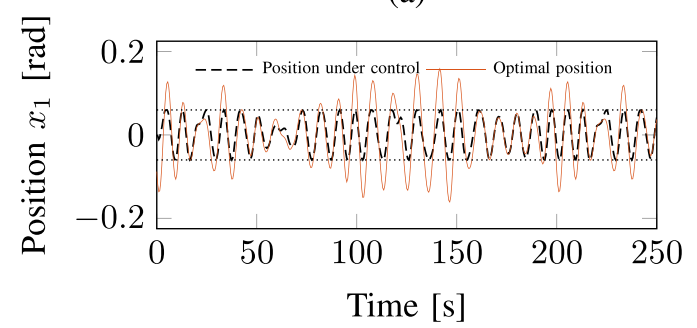

(b)

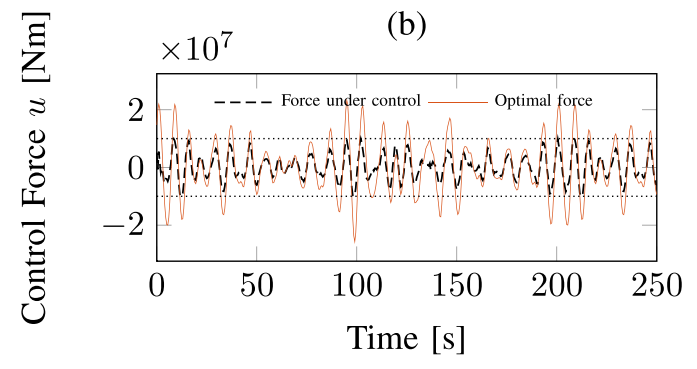

Fig. 8. Optimal trajectories (red solid line) and actual trajectories of the device under control (black dashed line). (a) Position under constraints (horizontal dotted lines) and (b) control force under constraints (horizontal dotted lines) for a 250 -s simulation.

simulation horizon, allowing its real-time application. Fig. 7 shows the optimal trajectory for the position and velocity of the device, and the optimal control force, while the black dashed curves represent the results from the real-time receding horizon control algorithm described in Section IV. The optimal trajectory can be theoretically calculated from the exact cancellation of the reactive terms in the equation of motion (20)
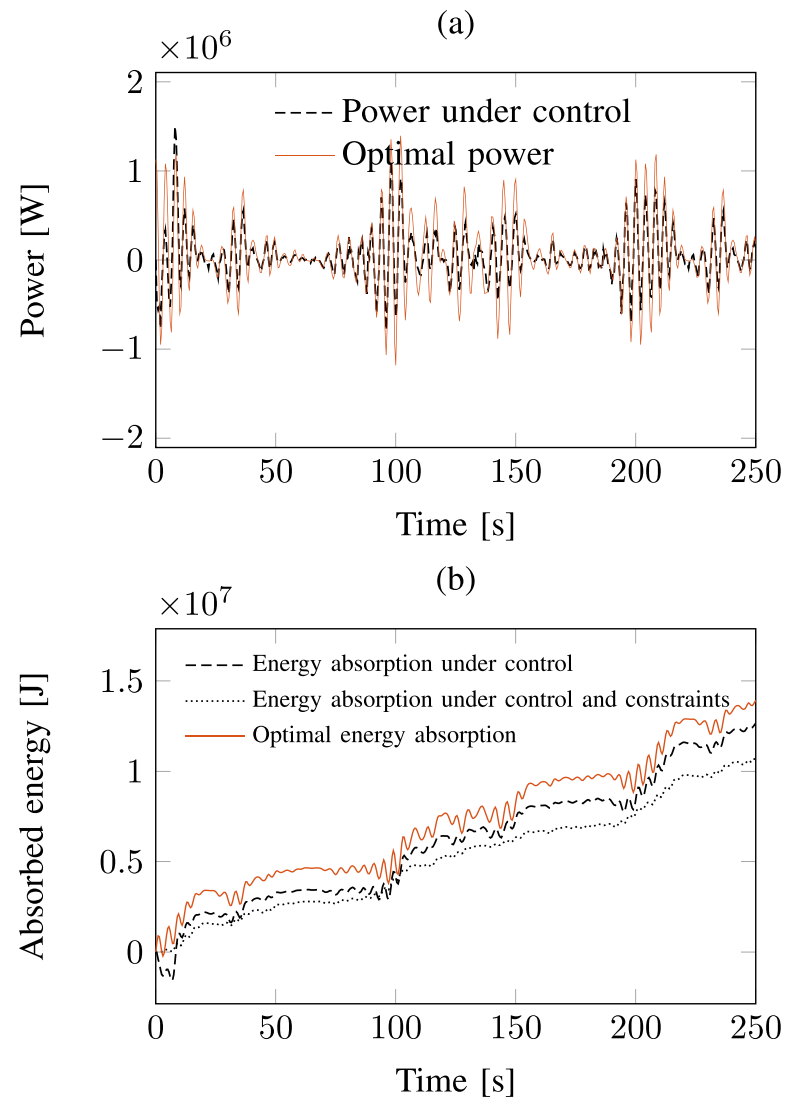

Fig. 9. Optimal trajectory (red solid line) and actual trajectories of the device under control (black dashed and dotted lines). (a) Absorbed power and (b) energy for a 250-s simulation.

by the optimal control force, i.e., stiffness, mass, and added mass terms [41]. In the optimal case, the device is brought into resonance for all frequencies and thus a small variation of the control force can induce large displacement differences. The velocity and control force determined using the pseudospectral method are seen to be close to the optimal solution from Fig. 7. However, some differences between the optimal and calculated position of the WEC, due to restoring force terms, are also evident. Nevertheless, the differences between the optimal trajectory and the actual position of the device under control do not significantly affect energy absorption, since the variations are of lower frequency than the wave excitation, and relate to (stored) potential energy that is not directly involved in the energy absorption. Fig. 8 illustrates the ability of the control algorithm to handle path constraints.

Fig. 9 presents the optimal absorbed power and the maximal energy absorbed by the WEC, and under control. Despite variations from the optimal trajectory, specifically for the position and the control force, the control gives a large absorption rate, and the total efficiency reaches $98.2 \%$ (excluding the initial transient), meaning that almost all the available energy is recovered.

\section{Conclusion}

The application of pseudospectral methods for the real-time receding horizon energy maximization problem requires the careful choice of a basis function that can cater for both transient and steady-state response components. In particular, 
to approximate nonperiodic functions, specific adjustments need to be made, for example, using cutoff functions to ensure periodicity. HRCF functions complement Fourier analysis and satisfy the nonperiodicity requirement without introducing bias, via the direct transformation of the input variable, and some additional computation, since HRCF functions are directly implementable in the pseudospectral approach.

The method described in this paper is adjustable in computational complexity in order to match real-time requirements. The number of basis functions employed and the number of iterations in the optimization process constitute variables that have to be adapted to the control hardware limitations. The number of basis functions corresponds to the dimension of the space where the optimization algorithm searches for a solution; hence, the computational time can be reduced if the solution depends only on few variables. Pseudospectral methods present a pragmatic approach to solve optimal control problems and allow path limitations that ensure safety and durability of the WEC to be taken into account.

The solution developed in this paper suits renewable energy systems, where the objective is to maximize converted energy, subject to the retention of stability and adherence to physical system constraints. In particular, the HRCF basis function set is well suited to the wave energy control problem, where the excitation force and system variables are well modeled using harmonic signals. The example case study shows that the developed real-time controller can achieve energy capture rates approaching the theoretical optimum for a flap-type WEC.

\section{APPENDIX}

\section{Convolution Product}

Cummins' equation (20) involves the determination of a convolution product in order to estimate part of the radiation force acting on the device hull. The convolution product between the scaled velocity $x_{2}$ of the device and the scaled radiation kernel function $k$ is split into two terms $c_{0}$ and $c$

$$
\begin{aligned}
L(\tau) & =\int_{g^{-1}(0)}^{\tau} k\left(\tau-s+g^{-1}(0)\right) x_{2}(s) d s \\
& =c_{0}(\tau)+c(\tau)
\end{aligned}
$$

where

$$
\left\{\begin{array}{l}
c_{0}(\tau)=\int_{g^{-1}(0)}^{-1} k\left(\tau-s+g^{-1}(0)\right) x_{2}(s) d s \\
c(\tau)=\int_{-1}^{\tau} k\left(\tau-s+g^{-1}(0)\right) x_{2}(s) d s .
\end{array}\right.
$$

For $s \in\left[g^{-1}(0),-1\right], x_{2}$ corresponds to past values of the velocity and is thus known. The first part of the convolution product $c_{0}$ is directly computed based on past velocity values. Replacing the state variable $x_{2}$ in the second term $c$, by its HRCF truncated series, defined in the following, we get:

$$
x_{2}(\tau)=\sum_{k=0}^{N} \phi_{k}(\tau) x_{2 k}=\Phi(t) X_{2}
$$

and

$$
c(\tau)=\sum_{k=0}^{N} c_{k}(\tau) x_{2 k}
$$

with

$$
c_{k}(\tau)=\int_{-1}^{\tau} k\left(\tau-s+g^{-1}(0)\right) \phi_{k}(s) d s .
$$

The orthogonal projection of $c_{k}$ over each basis function $\phi_{j}$ is $p_{k j}$ and defined by the following:

$$
p_{k j}=\int_{-1}^{1} c_{k}(\tau) \phi_{j}(\tau) d \tau \text {. }
$$

Finally, the matrix $P=\left[p_{k j}\right]_{k, j}$ is used to define the radiation force, so that

$$
\begin{aligned}
L(\tau) & =c_{0}(\tau)+\sum_{k=0}^{N} \sum_{j=0}^{N} \phi_{j}(t) p_{k j} x_{2 k} \\
& =c_{0}(\tau)+\Phi(\tau) P X_{2} .
\end{aligned}
$$

The expression for the convolution term is replaced in the expression of the equality constraints (21), i.e., inside the function $\mathbf{f}$.

\section{REFERENCES}

[1] Y. Hong, R. Waters, C. Boström, M. Eriksson, J. Engström, and M. Leijon, "Review on electrical control strategies for wave energy converting systems," Renew. Sustain. Energy Rev., vol. 31, pp. 329-342, Mar. 2014.

[2] F. O. Rourke, F. Boyle, and A. Reynolds, "Tidal energy update 2009," Appl. Energy, vol. 87, no. 2, pp. 398-409, 2010.

[3] S. Behera, S. Sahoo, and B. B. Pati, "A review on optimization algorithms and application to wind energy integration to grid," Renew. Sustain. Energy Rev., vol. 48, pp. 214-227, Aug. 2015.

[4] W. Qi, J. Liu, and P. D. Christofides, "Supervisory predictive control for long-term scheduling of an integrated wind/solar energy generation and water desalination system," IEEE Trans. Control Syst. Technol., vol. 20, no. 2, pp. 504-512, Mar. 2012.

[5] B. Teillant, R. Costello, J. Weber, and J. Ringwood, "Productivity and economic assessment of wave energy projects through operational simulations," Renew. Energy, vol. 48, pp. 220-230, Dec. 2012.

[6] A. Verbruggen et al., "Renewable energy costs, potentials, barriers: Conceptual issues," Energy Policy, vol. 38, no. 2, pp. 850-861, 2010.

[7] L. Y. Pao and K. E. Johnson, "Control of wind turbines," IEEE Control Syst., vol. 31, no. 2, pp. 44-62, Apr. 2011.

[8] J. V. Ringwood, G. Bacelli, and F. Fusco, "Energy-maximizing control of wave-energy converters: The development of control system technology to optimize their operation," IEEE Control Syst., vol. 34, no. 5, pp. 30-55, Oct. 2014.

[9] S. E. Ben Elghali, M. E. H. Benbouzid, and J.-F. Charpentier, "Modelling and control of a marine current turbine-driven doubly fed induction generator," IET Renew. Power Generat., vol. 4, no. 1, pp. 1-11, Jan. 2010.

[10] D. Garg, W. W. Hager, and A. V. Rao, "Pseudospectral methods for solving infinite-horizon optimal control problems," Automatica, vol. 47, no. 4, pp. 829-837, 2011.

[11] G. Bacelli and J. V. Ringwood, "Numerical optimal control of wave energy converters," IEEE Trans. Sustain. Energy, vol. 6, no. 2, pp. 294-302, Apr. 2015.

[12] G. Bacelli and J. Ringwood, "Constrained control of arrays of wave energy devices," Int. J. Marine Energy, vols. 3-4, pp. e53-e69, Dec. 2013.

[13] P. Williams, "Application of pseudospectral methods for receding horizon control," J. Guid., Control, Dyn., vol. 27, no. 2, pp. 310-314, 2004.

[14] F. Fahroo and I. M. Ross, "Pseudospectral methods for infinite-horizon nonlinear optimal control problems," J. Guid., Control, Dyn., vol. 31, no. 4, pp. 927-936, 2008.

[15] D. Huybrechs, "On the Fourier extension of nonperiodic functions," SIAM J. Numer. Anal., vol. 47, no. 6, pp. 4326-4355, 2010.

[16] H. Fu and C. Liu, "A buffered Fourier spectral method for non-periodic PDE," Int. J. Numer. Anal. Model., vol. 9, no. 2, pp. 460-478, 2012.

[17] B. A. Finlayson and L. E. Scriven, "The method of weighted residualsA review," Appl. Mech. Rev., vol. 19, no. 9, pp. 735-748, 1966.

[18] J. Vlassenbroeck, "A Chebyshev polynomial method for optimal control with state constraints," Automatica, vol. 24, no. 4, pp. 499-506, 1988. 
[19] C. A. J. Fletcher, Computational Galerkin Methods. New York, NY, USA: Springer, 1984.

[20] R. D. Russell and L. F. Shampine, "A collocation method for boundary value problems," Numer. Math., vol. 19, no. 1, pp. 1-28, 1972

[21] Y. Nesterov and A. Nemirovskii, Interior-Point Polynomial Algorithms in Convex Programming, vol. 13. Philadelphia, PA, USA: SIAM, 1994.

[22] H. R. Marzban and M. Razzaghi, "Rationalized Haar approach for nonlinear constrained optimal control problems," Appl. Math. Model., vol. 34, no. 1, pp. 174-183, 2010.

[23] A. K. Dizicheh, F. Ismail, M. T. Kajani, and M. Maleki, "A Legendre wavelet spectral collocation method for solving oscillatory initial value problems," J. Appl. Math., vol. 2013, Apr. 2013, Art. no. 591636.

[24] P. S. Addison, The Illustrated Wavelet Transform Handbook: Introductory Theory and Applications in Science, Engineering, Medicine and Finance. New York, NY, USA: Taylor \& Francis, 2002.

[25] I. M. Ross and F. Fahroo, "Legendre pseudospectral approximations of optimal control problems," in New Trends in Nonlinear Dynamics and Control and Their Applications. Heidelberg, Germany: Springer-Verlag, 2003, pp. 327-342.

[26] F. Fahroo and I. M. Ross, "Direct trajectory optimization by a Chebyshev pseudospectral method," J. Guid., Control, Dyn., vol. 25, no. 1, pp. 160-166, 2002

[27] P. Williams, "Jacobi pseudospectral method for solving optimal control problems," J. Guid., Control, Dyn., vol. 27, no. 2, pp. 293-297, 2004.

[28] J. F. Epperson, "On the Runge example," Amer. Math. Monthly, vol. 94, no. 4, pp. 329-341, 1987.

[29] J. A. Cretel, G. Lightbody, G. P. Thomas, and A. W. Lewis, "Maximisation of energy capture by a wave-energy point absorber using model predictive control," in Proc. 18th IFAC World Congr., Milan, Italy, Aug. 2011, pp. 3714-3721.

[30] M. J. D. Powell, "A fast algorithm for nonlinearly constrained optimization calculations," in Numerical Analysis. Berlin, Germany: Springer, 1978, pp. 144-157.

[31] P. V. Kokotović, "The joy of feedback: Nonlinear and adaptive," IEEE Control Syst., vol. 12, no. 3, pp. 7-17, Jun. 1992.

[32] B. Orel and A. Perne, "Chebyshev-Fourier spectral methods for nonperiodic boundary value problems," J. Appl. Math., vol. 2014, Jun. 2014, Art. no. 572694, doi: 10.1155/2014/572694.

[33] B. Orel and A. Perne, "Computations with half-range Chebyshev polynomials," J. Comput. Appl. Math., vol. 236, no. 7, pp. 1753-1765, 2012

[34] T. Pérez and T. I. Fossen, "Time- vs. frequency-domain identification of parametric radiation force models for marine structures at zero speed," Model., Identificat. Control, vol. 29, no. 1, pp. 1-19, 2008.

[35] G. R. B. Prony, "Essai experimental et analytique, etc," J. l'École Polytechn., vol. 1, no. 2, pp. 24-76, 1975.

[36] M. T. Pontes, "Assessing the European wave energy resource," J. Offshore Mech. Arctic Eng., vol. 120, no. 4, pp. 226-231, 1998.

[37] A. F. de O. Falcão, "Wave energy utilization: A review of the technologies," Renew. Sustain. Energy Rev., vol. 14, no. 3, pp. 899-918, 2010.

[38] F. Fusco and J. V. Ringwood, "A study of the prediction requirements in real-time control of wave energy converters," IEEE Trans. Sustain. Energy, vol. 3, no. 1, pp. 176-184, Jan. 2012.

[39] F. Fusco and J. V. Ringwood, "Short-term wave forecasting for real-time control of wave energy converters," IEEE Trans. Sustain. Energy, vol. 1, no. 2, pp. 99-106, Jul. 2010.

[40] K. Budal and J. Falnes, "Interacting point absorbers with controlled motion," in Power From Sea Waves, B. Count, Ed. London, U.K.: Academic, 1980, pp. 381-399.

[41] J. Falnes, Ocean Waves and Oscillating Systems: Linear Interactions Including Wave-Energy Extraction. Cambridge, U.K.: Cambridge Univ. Press, 2002.

[42] G. Li and M. R. Belmont, "Model predictive control of sea wave energy converters-Part II: The case of an array of devices," Renew. Energy, vol. 68, pp. 540-549, Aug. 2014.
[43] W. E. Cummins, "The impulse response function and ship motions," DTIC, David Taylor Model Basin, Washington, DC, USA, Tech. Rep. DTMB-1661, 1962

[44] G. Li, "Nonlinear model predictive control of a wave energy converter based on differential flatness parameterisation," Int. J. Control, vol. 89 , pp. 1-10, 2015, doi: 10.1080/00207179.2015.1088173.

[45] Y. Choi and W. K. Chung, PID Trajectory Tracking Control for Mechanical Systems (Lecture Notes in Control and Information Sciences), vol. 298. Springer Publishing Co., 2004.

[46] V. Utkin, J. Guldner, and J. Shi, Sliding Mode Control in ElectroMechanical Systems, vol. 34. Boca Raton, FL, USA: CRC Press, 2009.

[47] S. M. Rozali, M. F. Rahmat, and A. R. Husain, "Backstepping design for position tracking control of nonlinear system," in Proc. IEEE Int. Conf. Control Syst., Comput. Eng. (ICCSCE), Nov. 2012, pp. 77-82.

[48] M. Folley, T. Whittaker, and J. Van't Hoff, "The design of small seabed mounted bottom hinged wave energy converters," in Proc. 7th Eur. Wave Tidal Energy Conf., vol. 455. Porto, Portugal, 2007, pp. 1-10.

[49] G. Delhommeau, "Seakeeping codes AQUADYN and AQUAPLUS," in Proc. 19th WEGEMT School Numer. Simulation Hydrodyn., Ships Offshore Struct., 1993.

[50] C. L. Bretschneider, "Wave variability and wave spectra for windgenerated gravity waves," Beach Erosion Board, U.S. Army Corps Eng., Washington, DC, USA, Tech. Rep. 118, 1959.

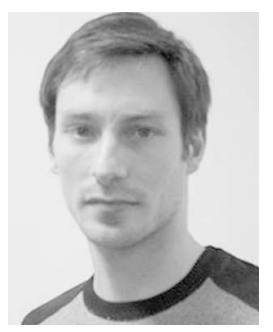

Romain Genest received the bachelor's degrees in mechanics and electronics from the École Normale Supérieure de Rennes, Rennes, France, the master's degree in hydrodynamics and the Ph.D. degree in mechanics from the École Centrale de Nantes, Nantes, France, and the Agregation diploma in engineering sciences to teach in French Preparatory School. His Ph.D. thesis concerned the development and experimental validation of control strategies for wave energy converters.

$\mathrm{He}$ is currently a Post-Doctoral Researcher with the Centre for Ocean Energy Research, Maynooth University, Maynooth, Ireland, where he focuses on the control of wave energy devices.

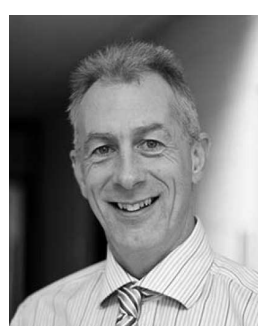

John V. Ringwood (M'87-SM'97) received the Diploma degree in electrical engineering from the Dublin Institute of Technology, Dublin, Ireland, and the $\mathrm{Ph} . \mathrm{D}$. degree in control systems from Strathclyde University, Glascow, U.K.

He was with the School of Electronic Engineering, Dublin City University, Dublin, and has held visiting positions with Massey University, Palmerston North, New Zealand, and The University of Auckland, Auckland, New Zealand. He is currently the Professor of Electronic Engineering and the Director of the Centre for Ocean Energy Research with Maynooth University, Maynooth, Ireland. He is a Chartered Engineer.

Dr. Ringwood is also a fellow of Engineers Ireland. 